\title{
A Decentralized Approach to Education Powered by Blockchain Technology
}

\author{
Suna Kyun ${ }^{1}$, Jaekyung $\mathrm{Yi}^{2}$, Jiyoung Jang ${ }^{3}$ \\ ${ }^{1}$ Assistant Professor, Devision of General Education, Sookmyung Women's University, South Korea, \\ skyun@sookmyung.ac.kr \\ ${ }^{2}$ Professor, Devision of Education, Sookmyung Women's University, South Korea,, \\ jklee@sookmyung.ac.kr \\ ${ }^{3}$ Collaboration professor, Center for Industry-Coupled Problem-Based Learning, \\ Hanyang University, South Korea, edujyjang@hanyang.ac.kr
}

Corresponding author: Jiyoung Jang

\begin{abstract}
Recently, some researchers in the field of education have been interested in the applications of Blockchain technology. While some researchers have conducted on the 'academic certificate handling' or 'managing record-keeping' in which are detailed educational administrative sector, others are more interested in reshaping educational landscape based on the key concept of 'decentralization' of Blockchain technology in a larger educational context. Researchers believe that the current centralized learning system or educational model is no longer sustainable, and that the Blockchain technology allows for a total disintermediation and disaggregation of education, specifically in higher education including lifelong learning. In a similar context, this paper will explore how the concept of 'decentralization', which is core concept of Blockchain technology, can transform the landscape of education, specifically focusing on the suggestions of researchers at Knowledge Media Institute at the open university in the UK. In other words, it will investigate how this new paradigm of Blockchain technology can affect on educational domain. To achieve this purpose, firstly, the basic Blockchain technology will be scrutinized, followed by the current major research outcome of educational applications of Blockchain technology will be reviewed. Finally, the implications and future prospects of educational applications of the Blockchain technology will be discussed.
\end{abstract}

Keywords: Blockchain Technology, Decentralisation, Higher Education, Lifelong Learning, Online Education

\section{Introduction}

When it comes to Blockchain, it reminds people of cryptocurrency as Blockchain technology is the core technology of cryptocurrency and we often refer to the same image as the main character of cryptocurrency[1]. To be exact, Blockchain is a technology that underlies cryptocurrency.

Meanwhile, Blockchain is continually being reported as a new technology that will lead the 4th industrial revolution as the key technology for future industries in the 21st century. According to World Economic Forum (after, WEF) in 2016, Blockchain was selected as one of the key technologies to lead the 4th industrial revolution era along with artificial intelligence(after, AI), Internet of Things(after, IoT) etc. Also, WEF predicted that $80 \%$ of the world's GDP will come from Blockchain-based technology by

Received: February 12, 2021; $1^{\text {st }}$ Review Result: March 29, 2021; $2^{\text {nd }}$ Review Result: May 20, 2021 Accepted: June 30, 2021 
2025[2].

Moreover, according to Tapscott and Tapscott (2018)[3], 'cars' came out in the 19th century, 'PCs' and 'Internet' in the 20th century, and Blockchain in the 21st century as the device of changing the world. It was predicted that Blockchain technology will fundamentally change the existing internet. In addition, Blockchain is an unprecedentedly simple and revolutionary protocol that records the value in public ledger that cannot be changed, which enables transactions that guarantee anonymity and security. The framework underlying the Blockchain goes beyond the function of simple cryptocurrency, and Blockchain technology will provide an important framework for transforming the existing 'Internet of Information' into 'Internet of Value' based on strong anonymity and security.

Furthermore, Blockchain technology, combined with AI, IoT, and big data, which are currently mentioned as key technologies for the 4th industrial revolution, is expected to contribute to securing individuals' privacy and information reliability in the digital society[3]. Currently, Blockchain technology is the most actively applied in the financial area, but it is gradually expanding to various areas such as medical care, distribution logistics, energy, and public services. The purpose of this paper is to discuss how such Blockchain technology can be educationally applied. In particular, Bockchain technology is greatly expected to contribute to the innovation of education in the current situation where online-based learning is becoming common and technology-enhanced learning is becoming essential.

To do this, the remaining paper is organized into three sections. Section 2 will explore the basic Blockchain technology which contains the 'key concepts' of Blockchain technology, followed by the 'types of Blockchain' and 'Blockchain paradigm'. Section 3 will review the current research of educational applications of Blockchain, specifically focusing on the concept of 'decentralization'. Finally, the implications and future prospects of the Blockchain technology applications in education will be discussed in section 4 .

\section{Overview of Blockchain Technology}

\subsection{Some Key Concepts}

Blockchain is a specific type of an ever-growing list of records, called blocks, are linked together to form a chain[4]. This is why we call it 'Blockchain'. Blockchain is secured by cryptographic techniques and managed by a decentralized community over a peer-to-peer (after, P2P) network. Each member of the community distributively maintains the storage of Blockchain[5]. Also, the transactions of Blockchain are immutable. Once a block inserted on the Blockchain, it becomes permanent and can not be modified retroactively, not even by the authors. The main concepts of Blockchain technology are as follows.

\subsubsection{Decentralization}

Blockchain is a distributed ledger that provides a way for information to be recorded and shared by a network. Blockchain is a specific type of database and it does not store information in a central location[6]. It is stored on the network of computers. Whenever a new block is added to the end of a Blockchain, all computers on the network update their Blockchain to reflect the change. By spreading the information across a network, rather than storing it in one central database, Blockchain is difficult to tamper with [7].

\subsubsection{Distributed Ledger}

Distributed ledger is a record of decentralized entries with no central registry and a Blockchain is a linked list of blocks that contains ledger entries more commonly known as transactions. A copy of 
Blockchain is held by every participating member in a P2P network. Blocks are added through a process of consensus between members. The design of a Blockchain ensures that once entered, block's contents cannot be changed even by authors and this property of Blockchain makes the entries of distributed ledger trustworthy[8][9].

\subsubsection{Distributed Storage}

Distributed storage is a decentralized approach of storing data in one or multiple servers. HTTP (i.e., Hyper Text Transfer Protocol) is considered the biggest distributed database where peers can access particular data from anywhere in the world. However, HTTP is gradually becoming obsolete technology because of it's centralized nature. P2P file systems are being gradually took its place. Although P2P file system comes with a lot of advantages, it has fatal drawbacks such as 1) unstable downloading, 2)unverified publisher, 3) lack of incentive mechanism, and 4) restricted its use. However, the emergence of Blockchain technology, that is, a combination of the distributed file system(i.e., P2P system) and Blockchain, is expected to solve all of these problems[5]. IPFS (InterPlanetary File System)[10], Swarm[11], and FileCoin[12] are some examples of such distributed storage.

\subsubsection{Security, Reliability, and Transparency}

Blockchain is a key technology that can decentralize existing centralized systems. Such distributed storage techniques ensure the security, reliability, and transparency of Blockchain data. In a Blockchain, data is written to a block, and the location is distributively stored in the computing power of each customer or organization, not on a particular central server[13].

Securiy : The current database structure by central system make expose everyone's information to the risk of hacking due to problems with server security itself or internal staff mistakes. The central system make the entire system stop, if a problem occur with central server. However the Blockchainbased distributed system can be permanently sustainable and free from the risk of hacking, unless the participants' systems on all networks are down[13][14].

Reliability : Block (i.e., information) on Blockchain is highly reliable because it is basically created by consensus from network users. In addition, Blockchain is based on P2P systems. The P2P system is a trust-based system and therefore it disappears when trust is lost. In other words, those who trust P2P systems participate in the system first. If the interaction results within the P2P systems are the same as they expected, they continue to participate in the system with greater trust. However, when they lose faith in the P2P system, they leave the system, and finally the whole system disappears[14]. Blockchain needs as many participants as possible because it needs to build trust through a number of agreements. Therefore, incentives are necessarily needed to gather participants, and cryptocurrency is used as an incentive. People become participants in the agreement to receive cryptocurrency, and Blockchain with participants creates a trust structure. In a nutshell, Blockchain uses consensus algorithms to ensure the reliability of the entire distributed system in an environment where distributed ledger is difficult to ensure reliability among participants.

Transparency : Due to the nature of Blockchain, anyone can use it for free, so all information is managed transparently. Anyone can access all transactions and events that occurred after the Blockchain was created, including the platform source code. In addition, blockchainized systems cannot be derecorded and falsified at all. Once information stored in blocks of a Blockchain system is irrevocable. Also, it cannot be tampered with or forged. Once registered in the system, the information is permanently stored and can be accessed by all participants[15]. 


\subsection{Blockchain Types}

Among various types of Blockchain, the major three Blockchains will be described in this section.

\subsubsection{Public Blockchain}

Public Blockchain has absolutely no access restrictions. Anyone with an internet connection can participate in transactions. The central system does not exist, and the system which participants are connected operates automatically. Cryptocurrency is the key to maintain such a public Blockchain system. That is, the system is operated according to the network user's participation without a central operator, network participants are paid cryptocurrency as compensation for their contribution to the agreement processes. The reason why we use public Blockchain is because of the safety of hacking which is the biggest advantage of this Blockchain. Key consensus algorithms used to maintain public Blockchain are 'Proof-of-Work', 'Proof-of-Stake' etc [14][16][17].

\subsubsection{Private Blockchain}

Private Blockchain can only be used by those who have obtained prior permission. Basically, most of the information recorded on the Blockchain should be accessible to all participants. However, there might be information that should not be disclosed, or that should only be disclosed to some people[16]. This is why private Blockchain has been emerged. Because private Blockchain consists of a small number of participants, only guaranteed participants can access information, and so competitive consensus algorithms, i.e., 'Proof-of-Work', 'Proof-of-Stake', are not required like public Blockchain. Theoretically, private Blockchain might not be a Blockchain. Therefore, some people consider private Blockchain as an encrypted database. Nevertheless, the reason why private Blockchain is attracting attention is because it is faster and more efficient than public Blockchain[14].

\subsubsection{Consortium Blockchain}

The consortium Blockchain is similar to a private Blockchain, but the only permitted user groups can use it, not individuals. Consortium Blockchain has the advantages of both public and private Blockchains. It is sometimes referred to as a shared ledger or federated ledger because of multiple approved parties using it within a federated environment[5]. These Blockchains are operated by a group or consortium. A single institution cannot control Blockchain, and the institutions which have permission can share governance.

\subsection{Blockchain Paradigm}

Currently, the world is rapidly shifting from a centralized business method(i.e., TTP-based network) to a P2P business method(i.e., Blockchain-based network). We call this emerging method or system Blockchain paradigm(see [Fig. 1]). That is, Blockchain paradigm means a decentralized business in which multiple computers work together by the pre-agreed rules without a central organization, and data is shared on all computers without being concentrated on a central server[2].

The existing internet has allowed for the possibility of illegal copying of content or making false payments by copying financial transaction records. However, Blockchain-based internet not only makes it impossible illegal-copying and double-payment, but also enables direct value movement without a central organization. Due to these nature of Blockchain network, it seems highly to be used in various industrial fields. Blockchain technology is expecting to transform the existing 'Internet of Information' into 'Internet of Value' by providing a protocol of trust to the internet(see [Fig. 1])[3]. 


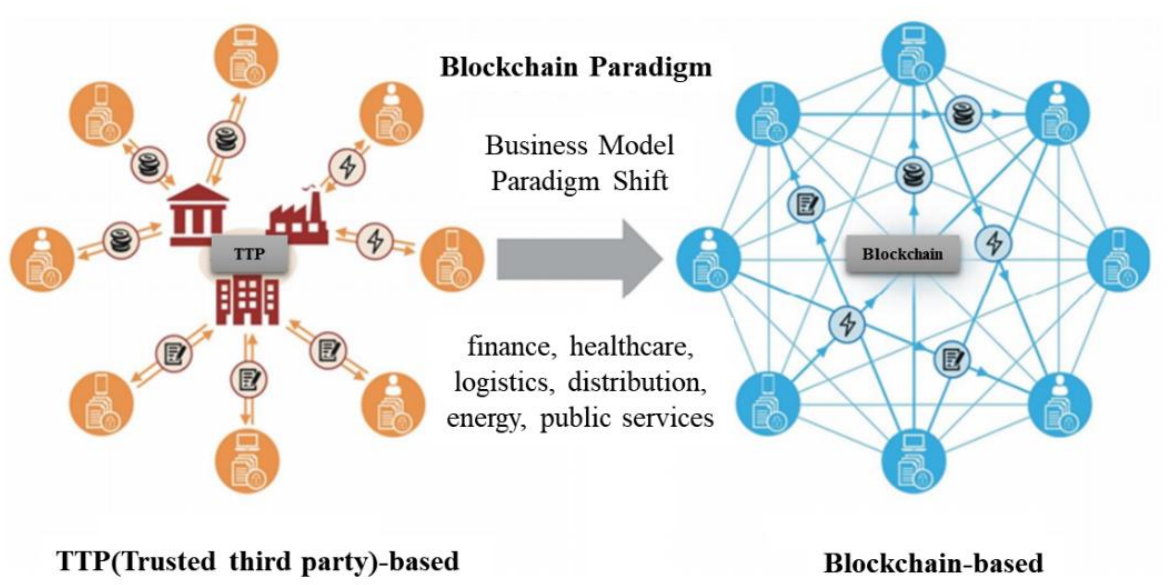

[Fig. 1] Blockchain Paradigm Shift :

From Trusted Third Party-based Business to Blockchain-based Business

(Source : KISTEP Technology Trends Brief 2018-1) [2]

\section{A Decentralized Approach to Education Using Blockchain Technology}

In this section, the recent research of how decentralization, the central concept of Blockchain technology[7], can be applied in education contexts will be discussed. Recently, researchers from Knowledge Media Institute(after, KMI) at the open university in the UK have been actively researching on the applications of Blockchain technology in higher education, including online-based lifelong learning. This section will discuss how Blockchain technology can be applied to the educational areas, focusing on KMI's current research outcomes and suggestions.

\subsection{Decentralized Educational Landscape}

In KMI's recent paper, Chowdhury, Ramachandran, Third, Mikroyannidis, Bacher, and Dominique (2020)[5] suggested a conceptual educational landscape based on Blockchain technology. The major concept was 'decentralization' of higher educational institutions. They indicated that current higher education is controlled mostly by isolated individual institutions which offer quality, credibility, governance, and administrative functions. According tho them, this centralized model of an isolated institution is not flexible enough, and therefore there are many difficulties in solving the problems faced by students. Hence, Chowdhury et al.[5] suggested a Blockchain-based decentralized educational model and explained this model can offer a passageway where educational institutions get to keep their individuality but participate in collaborations to help overcome the problems students face[5]. Also, they showed how higher educational institutions could potentially manage record-keeping, credential verification, and continued career support under the this decentralized educational environment.

\subsubsection{Current Educational Landscape and It's Problems}

The problems that all higher educational institutions around the world have in common, but that can be solved with Blockchain technology are as follows.

Problem 1 : Until now, all educational institutions maintained individual database of their own to store and hold students' records including their personal information. In most cases, students have no control over their own data and have no idea what kinds of their information the institutions have stored, while the institutions can control all data in their centralized databases if they want. This centralized 
database storage controlled by individual educational institutions can cause problems, specifically in this digital age. Most of all, such an approach can cause problems with the reliability of data. In this centralized storage system, the students' data can be altered for numerous reasons including updating by mistakes or corruption. Most importantly deliberate manipulation by the controlling administrators leading to tempering or removal of data without the students' agreement. Also, Needless to say, this kind of centralized system is always exposed to the risk of hacking. A Blockchain-based decentralized approach is expected to solve the current these problems such as data reliability, security, and individuals' privacy.

Problem 2: All higher educational institutions maintain a tradition of carrying trust through diplomas and degrees. Each institution provides education to the selected students and issues certificates, diplomas and degrees as the evidences. It used to work when there were fewer institutions, and people recognized the certificates or degrees issued by a specific university or other institutions. However, now is an era when paper certificates or degrees are no longer trusted because of the handy technology to create fake document. Currently, determining the authenticity of 'degrees', 'transcripts' and 'other educational records' issued by each institution has been becoming an important issue. These kinds of problems of current educational institutions can be solved by Blockchain technology. Because once the data inserted to the Blockchain, it is immutable. As long as the data is stored in the Blockchain, it cannot be manipulated retroactively, not even by the authors.

Problem 3 : The existing educational system is scattered, where educational institutions operate standalone failing to provide continued career support for their students. These days, students usually are educated at one or more educational institutions to develop their careers. Each institution supports students to get a job, but such events are limited to a few years after graduation. Furthermore, institutions generally have access to records and degree information that they provided only. This makes it very difficult for students, who have completed education in various institutions to obtain skills and knowledge, in order to manage their careers. This scattered educational eco-system and one institution's limitation to access to other educational institution prevent students from adequately assessing one's potentials, helping them to apply for the right job, and guiding them to their career paths[5]. These kinds of problems will be easily solved in the Blockchain-based decentralized educational environments. If students' academic records are stored on the Blockchain, and if a transparent disclosure system for anyone to access will be built, this problem can be solved.

\subsubsection{Proposed Educational Landscape}

Ultimately, the purpose of Blockchain-based decentralized educational system is to give students access to and authority over their data, in order to solve the problems which the existing educational institutions have. Blockchain-based decentralized systems are expected to benefit both students and institutions. While students will be able to manage their careers more conveniently, educational institutions will be able to operate their institutions more efficiently.

To solve the three problems identified in the previous section, Chowdhury et al.(2020)[5] suggested a Blockchain-based decentralized educational system(See [Fig. 2]). In their educational system, Chowdhury et al. proposed a four-layer architecture where Blockchain forms the first layer from the bottom. According to this system design, there are three layers such as data layer, verification layer, and support layer on the Blockchain layer. Problem 1, 2, and 3 discussed in the previous section represent three broad areas of the educational landscape and form the top three layers in their educational system design(See [Fig. 2]). 


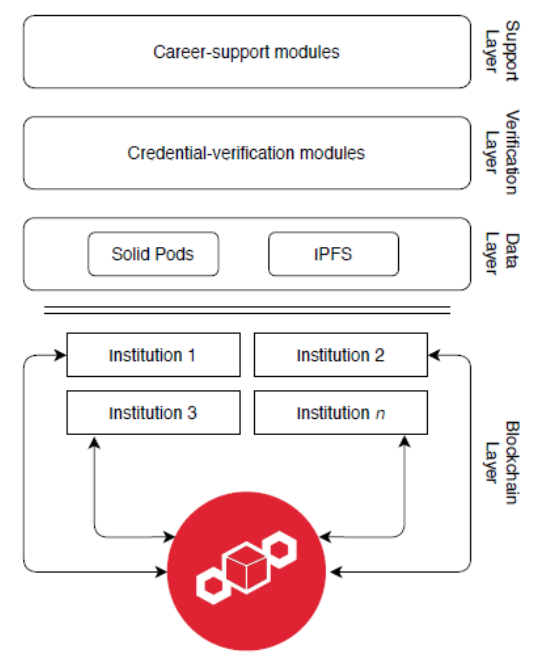

[Fig. 2] The Layered Architecutrue of the Blockchain-based

Proposed Decentralised Educational Landcape Suggested by Chowdhury et al.(2020)

(Sourse: Chowdhury, Ramachandran, Third, Mikroyannidis, Bacher, \& Dominique, 2020)[5]

According to Chowdhury et al.(2020)[5], they designed that Blockchain layer forms the foundation and adopted a consortium Blockchain among educational institutions. The remaining three layers will operate over this Blockchain and their specific details are as follows.

Data Layer is responsible for data governance and it manages students' data in a decentralized way. Data layer disintegrates the current central database of institutions and distributes its contents to various stakeholders such as students, instructors, and administrators of institutions. The data layer consists of IPFS and Solid (SOcial LInked Data) pods which are distributed storage. This data layer solves 'Problem 1 ' discussed in the previous section. It allows students to see what their educational institution holds on their behalf and also gives students control over their data. By using Blockchain, data layer also ensures the integrity of the information contained by students and administrators[5].

Verification layer is related to verify credentials. This layer helps students to get their qualifications checked for potential employers and other educational institutions. All institutions that confer degrees or award micro-degrees must give students a badge that students keep in their storage (i.e., Solid Pods in [Fig. 2]). Therefore, when individual students apply for courses in other institutions or jobs in companies, they can show the badge as an evidence of their qualification certificate.This verification layer solves 'Problem 2' discussed in the previous section. By making verification automated, it allows students to get their credentials verified at the expense of a few mouse clicks. It reduces time and saves money for both students and institutions who or which check their credentials[5].

Support layer is providing students career support. Both formal and non-formal educational institutions can participate in providing career support of students. These supports include suggesting jobs or courses, and preparing for automated CVs. The support layer solves 'Problem 3' discussed in the previous section. In a centralized and isolated system, educational institutions cannot see what qualification students obtained from other educational institutions in addition to theirs. However, in this proposed architecture by Chowdhury et al.(2020)[5], it is possible. Therefore institutions can come up with job and course suggestions to their students more precisely.

\subsection{Online-based higher Education and Lifelong Learning Powered by Blockchain Technology}

The Blockchain-based decentralized educational approach is very meaningful in this digital era when online-based higher education and lifelong learning are more and more becoming common. In this section, a decentralized approach for online-based higher education and also a learner-centered approach for lifelong learning powered by Blockchain technology will be discussed very briefly. 


\subsubsection{A Decentralised Approach for Online-Based Higher Education}

Recently, Mikroyannidis, Third, and Dominique(2020)[18] suggested a Blockchain-based decentralized educational model in online education (See [Fig. 3]). Though Mikroyannidis et al. called the model from [Fig. 3] online education, we believe that this model refers specifically to the onlinebased higher education model. Based on the model below, we will look at how decentralization, the core concept of Blockchain technology, can be practically implemented in the online-based higher education.

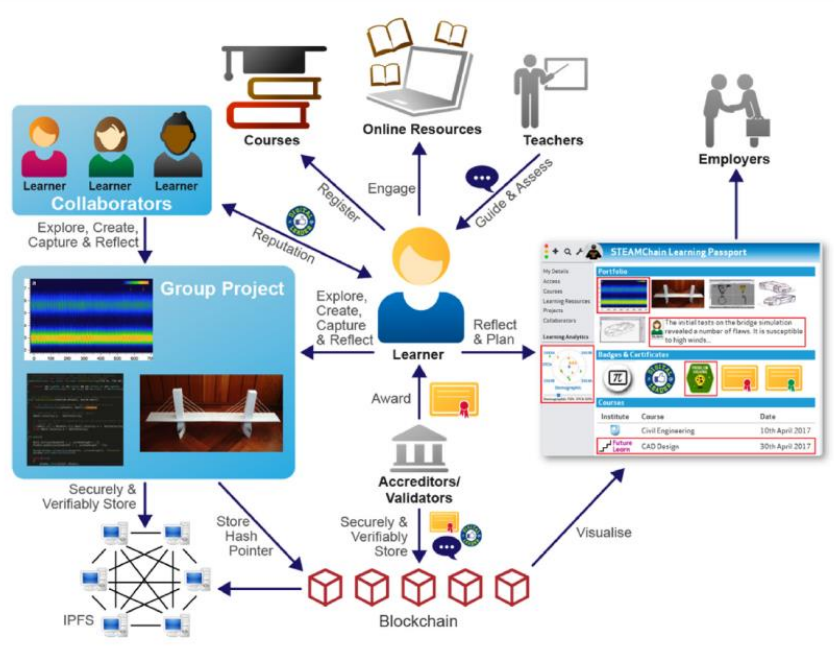

[Fig.3] A Blockchain-based Decentralized Approach to Online Education (source : Mikroyannidis, Third, \& Dominique, 2020)[18]

[Fig. 3] shows a visual representation of a Blockchain-based decentralized approach to online-based higher education. According to this model, students can register a number of courses which they have chosen, make use of additional learning resource, and receive feedback by tutors and other institutions staffs. Also, they can participate in group projects and can create shared project with their peer. The project results are stored in the way of IPFS(InterPlanetary File System, one of the examples of distributed storage) on the Blockchain. In addition to group project results, all data about students' accreditation, work, rating, formal and informal feedback are stored within a framework where everything is verifiable via the Blockchain[18]. Also, administrative departments of institutions issue formal certificates according to institutional processes using the data stored on the Blockchain when students request. Furthermore, the students' data stored on the Blockchain can be visually implemented in the form of e-portfolio which can be useful for students to find jobs or apply for another educational institution. As discussed in section 2, due to the nature of Blockchain technology, students' records generated by online network are highly reliable.

\subsubsection{Lifelong Learning with Blockchains}

Blockchain-based decentralized educational approach can be optimized for lifelong learning and lifelong learners. 'lifelong learning' means the learning which is not confined to childhood or the classroom. It can take place throughout life and lifelong learners pursue learning throughout their lifetime, both personal and professional reasons. They may study to acquire new skills that they need in their career or new job seeking. Also, they may study for personal reasons, such as a hobby of theirs. Therefore, lifelong learners may engage either formal or informal education, depending on their learning goals.

For these lifelong learners who face problems associated with the recognition of their learning achievements, Blockchain-based educational system can provide solutions. Once lifelong learner's 
achievements from either formal or informal education are stored on the Blockchain, this Blockchain based educational system can support lifelong learners in various ways. For example, this Blockchain based system can provide lifelong learners individualized recommendations about which job position might be suitable for them or what to study next[19]. In this way, Blockchain-based educational system aims to help lifelong learners reach their personal or professional learning goals. Mikroyannidis et al.(2020)[19] discussed and visualized this interaction between lifelong learners and educational institution in their paper (See [Fig. 4]).

[Fig. 4] shows how lifelong learners can get help from Blockchain-based educational system. That is, lifelong learners can study using various online courses provided by various either formal or informal educational institutions and all their learning results are stored on the Blockchain. Based on the stored data on the Blockchain, their achievements can be created in the form of e-portfolio which can be useful to find jobs or choose another educational course. Likewise, due to the nature of Blockchain technology, lifelong learners' records generated by online network are highly reliable.

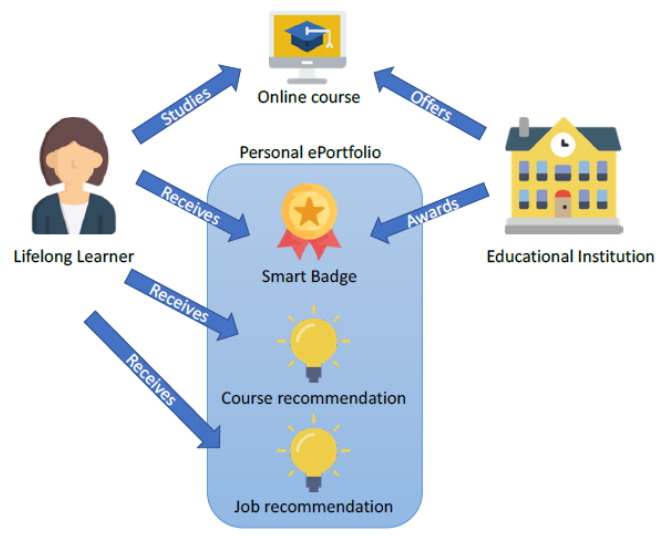

[Fig. 4] Stakeholder Interactions in the Main Scenrio of the Lifelong Learning Pilot (Source : Mikroyannidis, Third, Chowdhury, Bachler, \& Dominique, 2020)[19]

In section 3, we have explored the theoretical underpinning of how the basic concept of Blockchain technology, which has been scrutinized in section 2, can be applied educationally. Specifically it has been discussed, focusing on the the research outcome from KMI of open university in the UK.

In addition to the KMI research team, recently, many researchers, including the authors of this paper, are attempting related research. While Bozkurt and Ucar (2020)[20] investigated Blockchain technology as the bridging infrastructure in formal and non-formal or informal learning processes, Tugtekin, Dursun, and Ugur(2020)[21] examined the virtual identity in Blockchain. While De Bittencourt, Goedert, Sharma, and Bortolozzi(2020)[22] researched the framework of Blockchain in higher education, Nery, Macambira, Mota, and Rezende(2020)[23] explored the educational strategies between social media, cyberculture, Blockchain and education. Rodriques, Franco, Scheid, Kanhere, and Stiller (2020)[24] also researched on the Blockchain-based academic certificate handling.

Among the numerous detailed topics related to educational applications of Blockchain technology mentioned above, this study has scrutinized how decentralization, which is a core concept of Blockchain technology, might affect the educational domain, specifically online-based higher education or life-long learning. This can be said to be the purpose and significance of this paper.

\section{Discussion and Conclusion}

Currently, research on the applications of Blockchain technology in the field of education is in its early stages. While the research has been conducted in terms of applying major concepts of Blockchain 
technology to the current educational system and strategies so far, future research will require more specific research, such as how we design instructional eco-system based on the dynamics between learners, instructors and learning resources based on Blockchains.

According to the recent research, future higher educational institutions are expected to gradually shift from the current building-centered platform to a network-centered platform based on convergence of online and offline curriculum[25]. Perhaps, it will be a new higher educational system based on new technologies such as cloud computing, AI, and big data. Definitely, if Blockchain technology will be combined with this system, this system will be more powerfull. Meanwhile, AI and big data are still being actively used as tools for innovation of learning management systems and educational institutions. Likewise, if AI and big data are combined with Blockchain technology with the inherent nature of giving the data reliable, learning management systems and institutional management systems are expected to be stronger.

Most of all, if the concept of decentralization of Blockchain technology is smartly applied to the educational sector, it is expected that education and educational institutions might seem different from the present, but a truly learner-centered educational system will be established. That is the very networkcentered educational platform based on the integration of online and offline curriculum mentioned above, and the active application of Blockchain technology in the field of higher education will greatly contribute to make such system a reality.

In this study, we have mostly focused on online-based higher education and lifelong learning. However, a Blockchain-based decentralized approach to other educational fields, such as K12, early childhood education, will also greatly contribute to constructing an efficient system that allow all stakeholders to be happier. This system enable students (or, their parerents) to manage their data reliably and safely from the early childhood or elementary education. Also, they can also benefit from this reliable data, when they choosing a new course or learning activities. In addition to this, it can be used as a reliable evidence when they enter a new school. Teachers also can believe their newly admitted students' data because it is reliable due to the nature of Blockchain. Also teachers can manage their educational careers under the this system safely, reliably, and transparently. Most of all, this system enable each educational institution to reduce unnecessary efforts student record management, and therefore they can save money and also operate their institution more efficiently. In a nutshell, if a Blockchain-based decentralized approach is applied to the educational field, it will implement a system that satisfies all learners, teachers, and educational institutions regardless of school level.

\section{Acknowledgment}

This work was supported by National Research Foundation of Korea(NRF) grant funded by the Korea government(MSIT) (No. NRF 2020R1G1A1100739).

\section{References}

[1] Y. Lee, Future Technology, Informaion Publishing Group, (2019)

[2] G. You, K. Kim, KISTEP Technology Trends Brief 2018-1: Blockchain, Korea Institute of Science and Technology Evaluation, (2018)

[3] D. Tapscott, A. Tapscott, Blockchain Revolution: How the Technology Behind Bitcoin and Other Cryptocurrencies Is Changing the World, Portfolio, (2018)

[4] A. Mikroyannidis, A. Third, J. Domingue, M. Bachler, K, Quick, Blockchain Applications in Lifelong Learning and the Role of the Semantic Blockchain, Blockchain Technology Applications in Education, (2020), pp.16-41, DOI: 
https://doi.org/10.4018/978-1-5225-9478-9.ch002

[5] N. Chowdhury, M. Ramachandran, A. Third, A. Mikroyannidis, M. Bachler, J. Domingue, Towards A Blockchain-based Decentralised Educational Landscape, The Twelfth International Conference on Mobile, Hybrid, and On-line Learning, (2020), November 21-25, Valencia, Spain

[6] https://www.investopedia.com/terms/b/blockchain.asp, Mar 5 (2021)

[7] A. Grech, A. F. Camilleri, Blockchain in Education, JRC Science for Policy Report, European Commission, (2017)

[8] M. Ramachandran, N. Chowdhury, A. Third, J. Domingue, K, Quick, M. Bachler, Towards Complete Decentralised Verification of Data with Confidentiality: Different ways to connect Solid Pods and Blockchain, A Decentralised Web Workshop, (2020), April 20-24; Taipei, Taiwan

[9] N. Chowdhury, Inside Blockchain, Bitcoin, and Cryptocurrencies, (1st Edition), Auerbach, (2019)

[10] https://arxiv.org/abs/1407.3561 (J. Benet, "IPFS - Content Addressed, Versioned, P2P File System”), Mar 5, (2021)

[11] J. H. Hartman, I. Murdock, T. Spalink, The Swarm scalable storage system, the 19th IEEE International Conference on Distributed Computing Systems, (1999), June, Austin, TX, USA

[12] J. Benet, N. Greco, Filecoin: A decentralized storage network, Protocol Labs, Research, (2017)

[13] O. Park, Blockchain, Youngjin.com, (2019)

[14] Y. Park, A. Lian, S. Harmsen, Blockchain Revolution 2030, Kyobobook, (2019)

[15] S. Kim, Non-Destructive Trust Industrial Revolution Technology, Blockchain, Book Plus, (2018)

[16] M. Min, J. Choi, J. Jung, J. Kang, The Future of Blockchain, Digital Books, (2019)

[17] M. Jeon, Blockchain: Innovation of Gorvernmnet, Cloud Nine, (2019)

[18] A. Mikroyannidis, A. Third, J. Domingue, A case study on the decentralisation of lifelong learning using blockchain technology, Journal of Interactive Media in Education, (2020), Vol.2020, No.1, pp.1-10, DOI: http://doi.org/10.5334/jime. 591

[19] A. Mikroyannidis, A. Third, N. Chowdhury, M. Bachler, J. Domingue, Supporting Lifelong Learning with Smart Blockchain Badges, International Journal on Advances in Intelligent Systems, (2020), Vol.13, No.3\&4, pp.163-176.

[20] A. Bozkurt, H. Ucar, Blockchain Technology as a Bridging Infrastructure Among Formal, Non-formal, and Informal Learming Processes, Blockchain Technology Applications in Education, (2020), pp.1-15, DOI:10.4018/978-1-52259478-9.ch001

[21] E. B. Tugtekin, O. O. Dursun, S. S. Ugur, Virtual Identity in Blockchain, Blockchain Technology Applications in Education, (2020), pp.171-196, DOI: 10.4018/978-1-5225-9478-9.ch009

[22] D. F. Bittencourt, A. R. Goedert, R. C. Sharma, F. Bortolozzi, Framework Blockchain Education: Rupture in Higher Education, Blockchain Technology Applications in Education, (2020), pp.80-96, DOI: 10.4018/978-1-5225-94789.ch004

[23] M. B. M. Nery, M. O. Macambira, M. F. Mota, I. C. O. Rezende, Social Media, Cyberculture, Blockchains, and Education: A New Strategy for Brazilian Higher Education, Blockchain Technology Applications in Education, (2020), pp.242-259, DOI: 10.4018/978-1-5225-9478-9.ch012

[24] B. Rodriques, M. F. Franco, E. J. Scheid, S. S. Kanhere, B. Stiller, A Technology-driven Overview on Blockchainbased Academic Certificate Handling, Blockchain Technology Applications in Education, (2020), pp.197-223, DOI:10.4018/978-1-5225-9478-9.ch010

[25] S. Kyun, H. Jung, Y. Yang, D. Kang, S. Seo, Y, Kim, The Prospect of Distance and Higher Lifelong Education in the Fourth Industrial Revolution, Institute of Distance Educaton, Korea National Oen University, (2018) 